\title{
Surgical margins in the management of extremity soft tissue sarcoma
}

\author{
Makoto Endo ${ }^{1}$, Patrick P. Lin ${ }^{2}$ \\ ${ }^{1}$ Department of Orthopaedic Surgery, Kyushu University, Fukuoka, Japan; ${ }^{2}$ Department of Orthopaedic Oncology, MD Anderson Cancer Center, \\ Houston, TX, USA \\ Contributions: (I) Conception and design: All authors; (II) Administrative support: All authors; (III) Provision of study materials or patients: All \\ authors; (IV) Collection and assembly of data: All authors; (V) Data analysis and interpretation: All authors; (VI) Manuscript writing: All authors; (VII) \\ Final approval of manuscript: All authors. \\ Correspondence to: Patrick P. Lin. Department of Orthopaedic Oncology, MD Anderson Cancer Center, Houston, TX, USA.
}

Email: plin@mdanderson.org.

\begin{abstract}
Standard treatment of localized soft tissue sarcoma should include surgical resection, which can be performed alone or in combination with perioperative radiotherapy and/or chemotherapy. The purpose of surgical intervention is to excise the tumor completely and to prevent disease relapse. Surgeons should remove the tumor with a sufficiently wide margin of the surrounding normal tissue; however, it is also necessary to pay attention to maximizing postoperative physical function. In order to balance the two competing goals, surgeons have been working to establish better methods for determining surgical margins and better guidelines for achieving adequate margins. At the present time, limb-sparing surgery is the mainstay of surgical treatment for soft tissue sarcomas of the extremities. In this article, we review the fundamentals of surgical margins as they pertain to soft tissue sarcomas and make recommendations for surgical treatment based upon current literature.
\end{abstract}

Keywords: Surgery; margin; soft tissue sarcoma; extremity

Submitted Jul 14, 2018. Accepted for publication Aug 23, 2018.

doi: $10.21037 /$ cco.2018.08.10

View this article at: http://dx.doi.org/10.21037/cco.2018.08.10

\section{Major classifications of surgical margin}

Evaluation of surgical margin is important for the purposes of assessing the adequacy of the resection and informing clinicians with regard to the need for postoperative adjuvant therapy. A problem in the field of sarcoma treatment is that there are several classification systems for determining surgical margins, which means that the definition of positive or negative margin can vary between clinicians and centers. The classifications include notably the Enneking classification, the $\mathrm{R}$ classification, the Union International Contre le Cancer (UICC) classification $(\mathrm{R}+1 \mathrm{~mm}$ classification), dichotomous classification, and measurement of distance. These are currently the most popular classifications for surgical margin of soft tissue sarcoma (1).

\section{Enneking classification}

Enneking et al. proposed a system for the surgical staging of musculoskeletal sarcoma in 1980 (2). In his classification scheme, the four types of surgical margins were termed intralesional, marginal, wide, and radical (compartmental). The basis of the classification was the relationship of the margin to the tumor and its pseudocapsule. An intralesional margin would be obtained when the dissection passes within the lesion, which means the tumor's pseudocapsule has been opened and violated during surgery. Either macroscopic or microscopic tumor tissue is usually left at the margins, and there may be contamination of the exposed tissue planes. Most commonly, intralesional procedures are performed as a diagnostic incisional biopsy or by subtotal "debulking" resection of 
the tumor. A marginal margin results from a procedure in which the tumor is removed through the pseudocapsule or "reactive zone" around the tumor. When a marginal resection is performed for soft tissue sarcoma, there is a possibility of microscopic residual tumor. Marginal procedures are typically performed as an excisional biopsy, often by enucleation of the tumor. Sometimes resection with marginal margins are done when the tumor is located close to critical structures, including nerves, vessels, and organs. A wide margin is accomplished by a procedure in which the lesion is resected with the surrounding normal tissue. The plane of dissection is entirely through normal tissue but within the involved compartment. No effort is made to remove the entire length of involved muscle from origin to insertion. A wide resection is the standard procedure for extremity soft tissue sarcomas. A radical margin in achieved by a procedure in which the tumor is resected with wide margins plus the entire muscles and tissues in the anatomic compartment.

Enneking's classification is largely based upon the macroscopic findings during surgery. In evaluating the margins, the closest margin between the tumor and the resection surface should be assessed. That is, if the tumor is resected mostly with wide margins, but cut in the reactive area around the tumor even in a small part, the margin of this resection is judged marginal. Trovik et al. modified the Enneking's criteria to report adequate (radical or wide) or inadequate (marginal or intralesional) margins (3).

The Enneking classification system has been a popular method of evaluating surgical margin for soft tissue sarcoma because of its conceptual appeal and practical utility in surgical planning (1). However, reproducibility may be an issue. Trovik et al. reported that disagreement of margin assessment, especially between wide and marginal margins, occurred in 23 of the 117 randomly selected cases (19.7\%) (4).

\section{$R$ classification (the residual tumor classification)}

The $\mathrm{R}$ classification was adopted into the 3 rd edition of the AJCC Manual for Staging of Cancer (5). The R classification is based on both the macroscopic and microscopic assessment of the resection margins at the surgery. The margin is categorized either as grossly positive (R2), microscopically positive (R1), or microscopically negative (R0), which means that no tumor cells are seen at the inked resection surface with a microscope. The definition of each margin, R2, $\mathrm{R} 1$, and R0, is clear and easily understandable, so that the proportion of papers reporting surgical margins by the $\mathrm{R}$ classification has been increasing over time (1).

\section{UICC classification ( $R+1$ mm classification)}

In recent years, the $\mathrm{R}$ classification has evolved into the " $\mathrm{R}+1 \mathrm{~mm}$ " classification by the International Union Against Cancer (UICC), in which the margin is categorized as grossly positive (R2); for specimens to be considered microscopically negative, there had to be at least $1 \mathrm{~mm}$ of normal tissue between the tumor and the inked resection margin (R0), whereas specimens with tumor within 1 $\mathrm{mm}$ of the inked border were judged as microscopically positive (R1) (6). This means that the requirement for R0 margin in the UICC $(\mathrm{R}+1 \mathrm{~mm})$ classification is more stringent than that of the $\mathrm{R}$ classification. Currently, it is controversial which classification is the better predictor of local recurrence in soft tissue sarcoma $(7,8)$.

\section{Dichotomous classification (positive or negative)}

Some papers adopt the dichotomous classification, which reports only positive or negative margins (9). In most papers, positive margin means microscopically positive; however, it is not necessarily clear how many macroscopically positive cases are included in the positive margin group. There is a big difference between macroscopically and microscopically positive margins, so it is not recommended that this classification be used in scientific papers. For purposes of unambiguous communication, it is important to state whether positive margins mean macroscopically or microscopically positive.

\section{Measurement of the distance}

Some papers describe the measurement of the distance from the surface of the surgical resection to the edge of the tumor $(10,11)$. A potential problem of this method is that the distance between the resection surface and the tumor may vary under the influence of muscle shortening by formalin. A change in shape by inappropriate methods of fixation also causes a significant error in the assessment of distance. In data analysis, distance is a continuous variable, and its conversion to a categorical variable can become problematic since the cut-off values are different among the papers. This makes it difficult to compare the results across studies.

\section{Surgical margins and local recurrence}

It is widely accepted that surgical margin is related to the risk of local recurrence (Table 1) $(3,7,8,10-16)$. In one study 


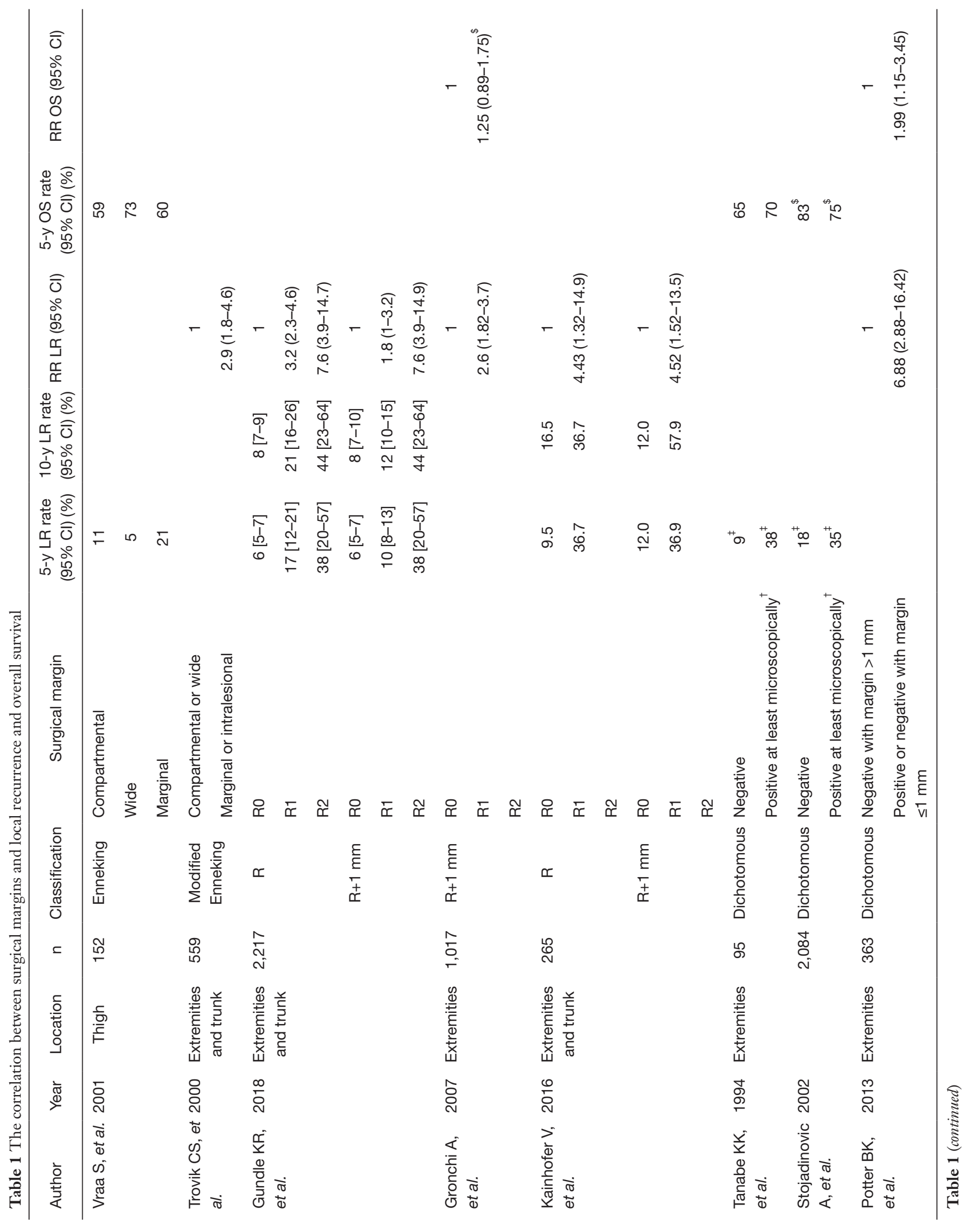




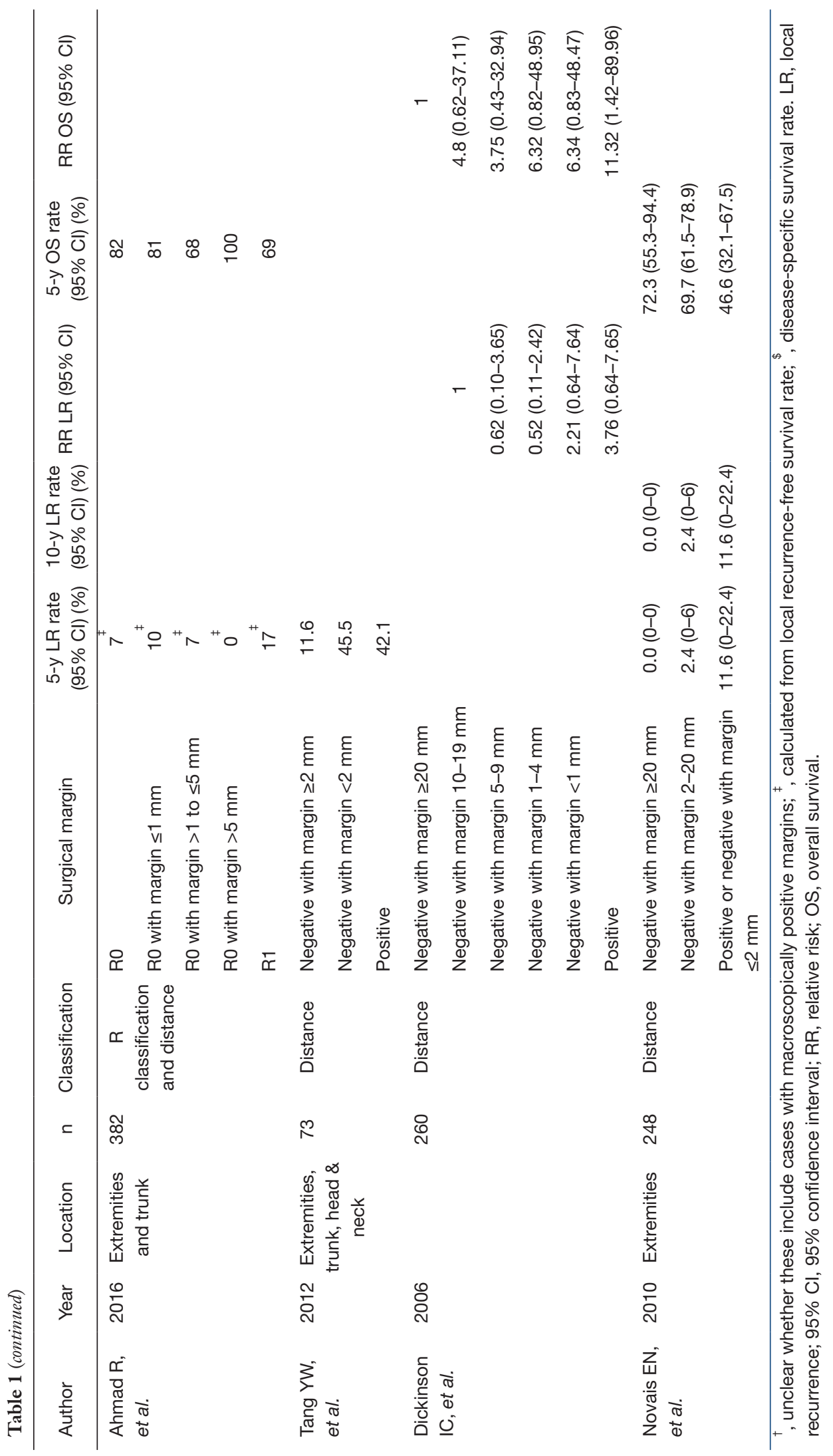


using Enneking's classification, the 5-year local recurrence rate was reported to be $11 \%, 5 \%$, and $21 \%$ with radical (compartmental), wide, and marginal resection of the soft tissue sarcoma of the thigh, respectively (12). There was a statistically significant difference in local recurrence rate between wide and marginal margins in that report (12). Additionally, in the multivariate analysis, surgical margin was found to be an independent prognostic factor for local recurrence (12). On the other hand, local recurrence rate of radical margin (excision of the entire anatomic compartment) was $11 \%$, which was higher than the $5 \%$ local recurrence rate of wide margin. Although the result seems counter-intuitive, it is likely that there was some form of selection bias in the study. Cases that were selected for radical resection were likely to have been larger and possibly affected by regional metastases or skip lesions.

With the $\mathrm{R}$ classification, the 5-year local recurrence rates were $6 \%, 17 \%$, and $38 \%$ with $\mathrm{R} 0, \mathrm{R} 1$, and $\mathrm{R} 2$ margin, respectively, in one study (8). In another paper, the 5-year local recurrence rate was reported $9.5 \%$ and $36.7 \%$ with R0 and R1 margins, respectively (7). These papers concluded that the risk of local recurrence could be estimated using the $\mathrm{R}$ classification.

It is not clear yet whether the UICC $(R+1 \mathrm{~mm})$ classification is superior to the original $\mathrm{R}$ classification in predicting local recurrence. In comparing the classification schemes, there are two important factors to keep in mind, namely the absolute value of the local recurrence rate and the difference in local recurrence rate between $\mathrm{R} 0$ and $\mathrm{R} 1$ resections. If surgery alone with $\mathrm{R} 0$ margins results in a low absolute value of the local recurrence rate, then no radiotherapy or other adjuvant therapy may be required. If surgery with R1 margins results in a substantially higher risk of local recurrence than $\mathrm{R} 0$ resection, then some sort of adjuvant therapy should be considered.

Gundle et al. retrospectively investigated 2,217 patients with non-metastatic extremity and axial soft tissue sarcoma treated with surgery and multidisciplinary modalities and reported that the 5-year local recurrence rate was $6 \%, 10 \%$, and $38 \%$ in patients treated by surgery with R0, R1, and R2 margins, respectively, using the UICC classification (8). When their cohort was re-analyzed using the R classification, the local recurrence rates for R0, R1, and $\mathrm{R} 2$ resections were $6 \%, 17 \%$, and $38 \%$, respectively. Local recurrence rate of $\mathrm{R} 1$ resection was higher in the $\mathrm{R}$ classification, whereas those of R0 resection was almost the same among the two classifications. The authors concluded that the $\mathrm{R}$ classification is more useful in the clinical setting because the UICC classification reduced the difference in local recurrence rate between $\mathrm{R} 0$ and $\mathrm{R} 1$, compared to the $\mathrm{R}$ classification. The results suggested that a negative margin with less than $1 \mathrm{~mm}$ width of the normal tissue might be adequate with multimodal treatment.

In contrast, Kainhofer et al. retrospectively studied 411 patients with soft tissue sarcoma, of which 265 patients were eligible for statistical analysis, and reported that the 10-year local recurrence rate was $16.5 \%, 12.0 \%, 36.7 \%$, and $57.9 \%$ with R0 (R classification), R0 (UICC classification), R1 (R classification), and R1 (UICC classification), respectively (7). Local recurrence rate of R0 resection using the UICC classification was lower than that of the $\mathrm{R}$ classification, whereas that of $\mathrm{R} 1$ resection was higher with UICC classification. They concluded that surgeons should plan a surgery to obtain R0 margin according to the UICC classification (minimal resection margin $>1 \mathrm{~mm}$ ) because the local recurrence rate was lower after $\mathrm{R} 0$ resection of the UICC classification compared to R0 resection of the $\mathrm{R}$ classification.

In general, the local recurrence rate of R0 resections is lower than that of R1 resections with both the R and UICC classifications. In a similar way, with measurement of the distance to assess margins, the local recurrence rate showed a tendency to be higher with closer distance between the surgical resection surface and the tumor $(10,11)$. However, it is not clear how exactly this might inform clinical decisions, such as the implementation of radiation boosts or re-resection of tumor beds.

\section{Surgical margins and overall survival}

It has been accepted that both macroscopic and microscopic positive surgical margins decrease local recurrence-free survival in soft tissue sarcomas of the extremities $(10,11,13)$. However, the influence of the surgical margin on overall or disease-specific survival is less clear. In Enneking's classification, there were no significant differences in overall survival rate between radical (compartmental) and marginal margins. The 5 -year overall survival rate was $59 \%$ vs. $60 \%$ for radical and marginal resections, respectively (12).

With regard to microscopic margins and survival, different results have been reported by various authors. Heslin et al. studied 168 patients with high-risk extremity soft tissue sarcoma and found that a positive microscopic margin was statistically associated with distant metastasis and tumor mortality (17). Similarly, Stojadinovic et al. investigated 2,084 adult patients with localized primary 
soft tissue sarcoma and showed that positive microscopic margins decrease distant recurrence-free survival rate and disease-specific survival (18). In contrast to these reports, Tanabe et al. found that in a group of 95 patients with extremity soft tissue sarcoma, negative surgical margins enhanced local disease control but did not improve overall survival (5-year overall survival rate; $65 \%$ vs. $70 \%$ for negative and positive margins, respectively) (19). Likewise, Bonvalot et al. studied 531 patients with primary extremity soft tissue sarcoma and reported that the margin status had no correlation to overall survival (20).

It is difficult to draw a strong conclusion based upon the current literature, and it is likely that more work will be needed in this area. If the effect is small, the number of patients in future studies will need to be large to have adequate power. Furthermore, consistency in the reporting of margins may need to be evaluated more critically.

\section{Soft tissue sarcomas of special considerations}

\section{Invasive soft tissue sarcoma}

Certain soft tissue sarcomas exhibit a highly infiltrative and invasive growth pattern, which may be manifested by the tail sign on MRI (21). An example of invasive soft tissue sarcoma would be myxofibrosarcoma, which tends to spread on the superficial fascial plane (22). The risk of local recurrence of myxofibrosarcoma is quite high and has been estimated to be approximately $20-30 \%$ (22-24). Haglund et al. found that local recurrences occurred in 11 of the 36 patients (31\%) (23). In this cohort, local recurrences were observed in 7 of the 27 patients (26\%) with negative margins and 4 of the 9 patients (44\%) with positive margins (23). It was interesting to note that among patients with negative microscopic margins, 6 of the 15 patients (40\%) with margins less than $1 \mathrm{~cm}$ developed local recurrences, whereas none of the 6 patients with $1 \mathrm{~cm}$ or greater margin had local recurrences. In another study on myxofibrosarcoma, Kikuta $e t a l$. described a group of patients for whom the surgical plan was to remove a margin of $>3 \mathrm{~cm}$ (22). Despite the intent to remove a generous margin in every patient, a histologically positive margin after surgery was evident in $28 \%$ of the cases. Local recurrence occurred in 21 of the 100 patients $(21 \%)$, and the estimated 5 -year recurrencefree survival rate was $74.8 \%$ (22).

Iwata et al. investigated the impact of infiltrative growth on the outcome of patients with undifferentiated pleomorphic sarcoma and myxofibrosarcoma (25). Their protocol for planning the extent of resection for invasive soft tissue sarcoma was to excise $2-3 \mathrm{~cm}$ from the edge of the tumor extension on imaging studies. Nevertheless, in spite of trying to remove a wide margin, the surgical margin was microscopically positive in 43 of the 89 patients $(48 \%)$ and the 5-year local failure-free survival rate was $81 \%$. These results collectively underscore the difficulty of achieving wide margins for infiltrative histologies. In order to obtain microscopically negative surgical margins, more than $3 \mathrm{~cm}$ margin on MRI images is potentially required in the planning of the operation in cases of infiltrative soft tissue sarcomas (Figure 1). The ideal margin of the excision for such tumors is unclear and remains to be established. The use of intra-operative frozen sections, particularly of the fascial margins, may be helpful as an additional guide during surgery for the extent of tissue removal.

\section{Atypical lipomatous tumor}

Atypical lipomatous tumor in the extremities is one of the less aggressive members of the adipocytic family of tumors. It has been called "well-differentiated liposarcoma" previously, but this appellation does not seem appropriate in the extremities since it does not have the same poor prognosis as similar tumors arising in the retroperitoneum. When it arises in the extremities, it shows no potential for metastasis unless it undergoes dedifferentiation. This observation led to the introduction of the term "atypical lipomatous tumor" for lesions arising at surgically amenable locations in the limbs and on the trunk (26). Atypical lipomatous tumor infrequently recurs after excision with a macroscopically clear margin, so that resection should strive to minimize surgical morbidity. En bloc complete resection is generally sufficient to obtain long-term local control, even if the tumor approaches the inked margins microscopically (27). In cases with multiple recurrences, radiation may be helpful in achieving control of the disease (28).

\section{Periosteal margins}

Soft tissue sarcomas sometimes occur near bones and may rest against the periosteal surface. In such cases, the presence of bone invasion should be assessed carefully on MRI and/or CT scan. Extension into bone has been reported to be a poor prognostic sign (29). Resection of the tumor with the adjacent bone should be considered if clear invasion into bone is demonstrated. According to one article with 50 cases of periosteal soft tissue sarcoma, true 

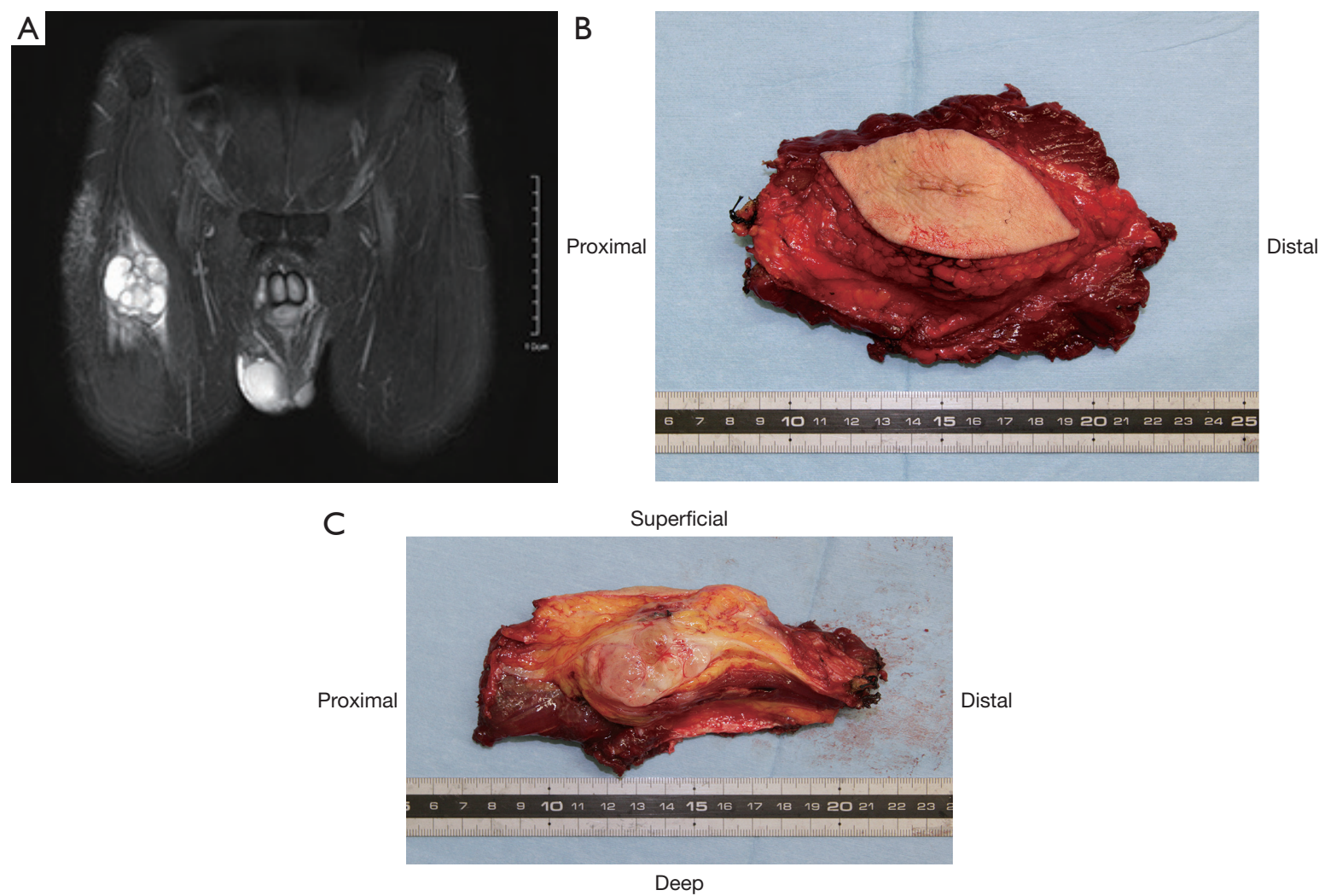

Figure 1 A case of myxofibrosarcoma of the right thigh is shown. Coronal T2-weighted fat-suppressed MRI shows a multinodular mass with high signal intensity in the right sartorius muscle with longitudinal extension of the high-intensity area (A). In surgery, the sartorius muscle is excised along the planned resection planes $7 \mathrm{~cm}$ apart from the tumor to completely remove the high-intensity area on MRI (B). The skin surrounding the biopsy scar as well as a part of the tensor fasciae latae and rectus femoris muscles are also excised. The cut surface shows a multinodular myxoid tumor with streaks spreading into the proximal and distal directions (C). Histopathological examination revealed the surgical margins to be negative microscopically.

invasion into the bone was confirmed by histopathologic examination in only 3 of the 50 cases (6\%) (30). In that study, local recurrence in the soft tissues developed in 8 of the 50 patients $(16 \%)$, however, there was no statistically significant difference in local recurrence rate between patients who had soft-tissue resection only and patients who had the adjacent bone resection at the same time. The authors concluded that relatively few soft tissue sarcomas can penetrate cortical bone and recommended composite bone and soft-tissue resections only for the tumor with frank bone invasion on images. It was the authors' opinion that the periosteum represented a firm barrier that was relatively resistant to penetration by soft tissue sarcoma. Treatment by wide resection, including subperiosteal dissection with Bovie electrocautery and radiation treatment to the bone, was considered adequate for most cases. In cases of extensive periosteal stripping and radiation to bone, there may be an elevated risk of a late radiation-associated fracture of the bone, which can be very difficult to heal (30). Prophylactic intramedullary fixation might be appropriate in some instances, particularly older women, patients receiving higher doses of radiation, and patients with low bone mineral density; however, this is a controversial subject, and it has not yet been shown that prophylactic nailing improves outcome.

\section{Soft tissue sarcomas of the hand}

Treatment of soft tissue sarcomas of the hand is a challenge to surgeons because of its complicated anatomy and the absence of reliable barriers, such as fascia, to separate anatomic compartments. Sacrificing tendons, blood 

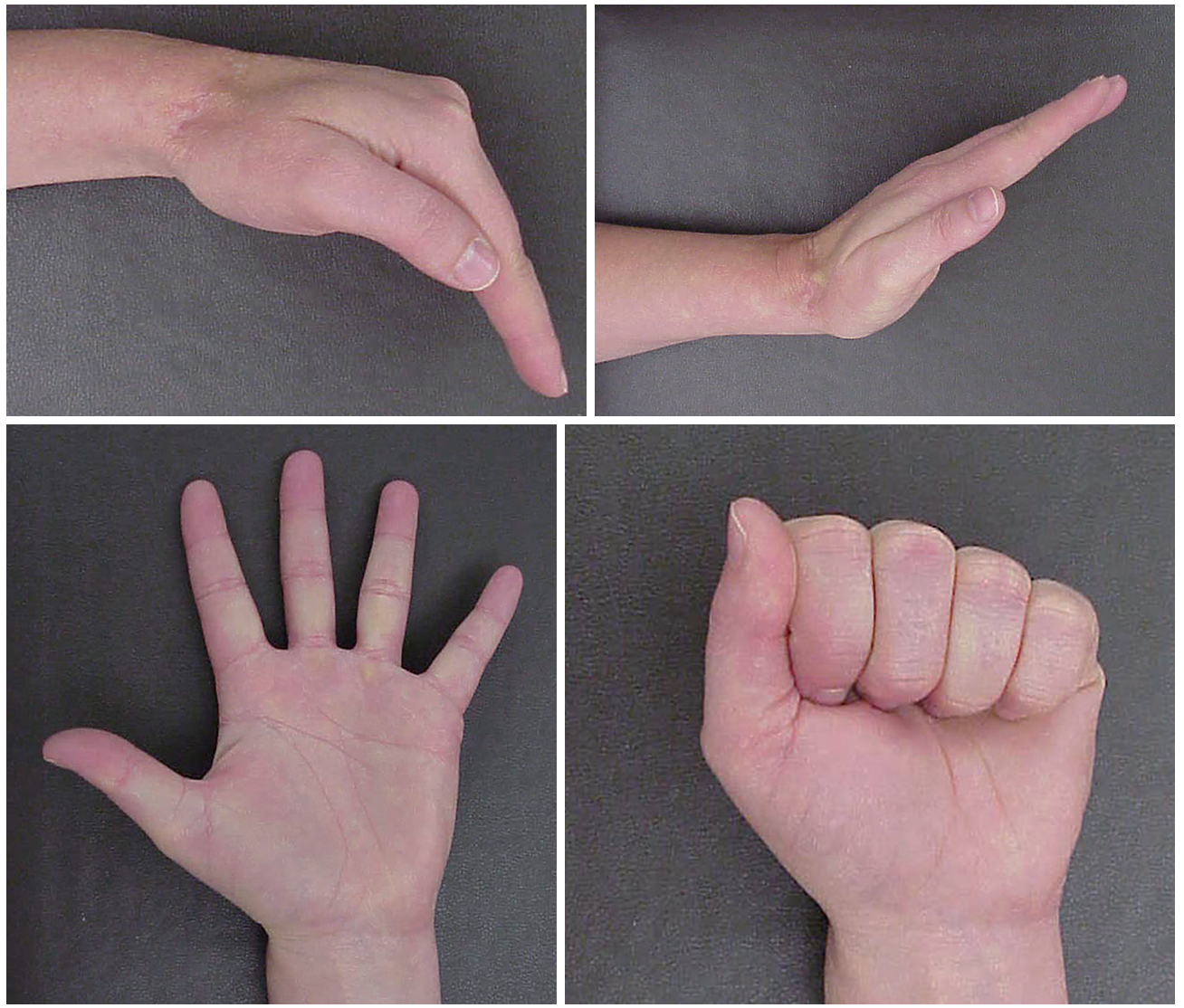

Figure 2 The range of motion of the wrist and hand is shown 1 year after 60 Gy post-operative radiation to the wrist and hand for epithelioid sarcoma in a 29 -year-old woman. There is some limitation of wrist dorsiflexion and palmar flexion, but she has unrestricted movement of the fingers and excellent grip strength.

vessels, and nerves to obtain adequate margins may result in significant functional loss. It is often difficult to obtain adequate margins in the resection of soft tissue sarcomas of the hand, and the rate of positive margin after surgery is reported to range from $11.3 \%$ to $36.4 \%$ (31-33). As is the case with other anatomic sites in the extremities, macroscopic positive margin is strongly associated with local recurrence of soft tissue sarcoma of the hand. The 5-year risk of local recurrence is $14 \%$ for wide or marginal margins and $80 \%$ for intralesional margin (33). Surgeons should avoid macroscopic positive margins; if it seems impossible to have macroscopic negative margins, amputation should be strongly considered. Of note, partial amputations of fingers, including ray amputations of multiple digits, can be compatible with good or even excellent function $(34,35)$. When margins are close or microscopically positive, adjuvant radiotherapy can be applied to selected cases, although radiotherapy does not completely negate the effect of positive margins (36). It is noteworthy that radiotherapy to the hand is related to an increased risk of complications and poor outcome (37-39). Radiotherapy to the hand may cause early complications such as wound dehiscence and wound necrosis, and late complications including ankylosis, joint contracture, adduction contracture, and osteitis (37). However, it is also important to note that radiotherapy is not contra-indicated in the hand, and when administered carefully, can still be associated with excellent long-term function (Figure 2). At certain centers, including MD Anderson Cancer Center, the preference for treating sarcomas of the hand that may have close margins is to deliver 50 Gy preoperative radiation. In most instances, the lower preoperative dose is well-tolerated and does not result in debilitating fibrosis.

In summary, whenever possible, aggressive surgery aiming at negative margins without radiotherapy, should be considered the preferred treatment strategy, and the surgeon 
should not refrain from performing ray amputations, especially of non-thumb digits. In cases where the margins will be close even with partial hand amputations, preoperative radiation with $50 \mathrm{~Gy}$ is compatible with good hand function, and some authors have concluded that surgery with adjuvant radiotherapy is acceptable treatment for patients with soft tissue sarcoma of the hand (40).

\section{Soft tissue sarcomas of the foot}

Soft tissue tumors of the foot are comparatively common; however, malignant ones are rare. The surgical considerations in the foot are somewhat similar to the hand in terms of the close proximity to critical structures such as nerves, vessels, and tendons as well as the paucity of expendable muscle and other connective tissue. One major difference is that the functional loss of movement in the digits and sensation does not carry the same functional significance as the hand. Consequently, soft tissue resections can be more aggressive in the foot than in the hand. Patients with excellent perfusion and collateral blood flow can do well after extensive resections, even if the plantar arteries and nerves are sacrificed with the tumor. In the modern era, limb-salvage surgery with or without radiotherapy is considered acceptable, mainstream treatment in the surgical management of soft tissue sarcomas of the foot, associated with an excellent local control and good functional outcome $(40,41)$. Cribb et al. retrospectively investigated the oncologic and functional outcome of 27 patients who had limb-sparing surgery for a soft tissue sarcoma of the foot or ankle and reported that all surviving patients were able to wear normal shoes and none required a walking aid (42). Because of the rarity of soft tissue sarcomas of the foot, these tumors are likely to be removed by inexperienced surgeons and unplanned surgery. Nishimura et al. raised awareness over unplanned surgery for sarcomas of the foot, which led to a high rate of local recurrence and a relatively worse prognosis (43). Since the function after below-knee amputation is excellent with modern prosthetics, surgeons should not hesitate to perform this operation if the margins are likely to be unacceptable with a limb-sparing operation.

\section{Lymph node metastasis and sentinel node biopsy}

Lymph node metastases in patients with soft tissue sarcomas are uncommon; however, some histologic subtypes are known to have a greater tendency of metastasizing to the lymph nodes. These would include rhabdomyosarcoma, clear cell sarcoma, epithelioid sarcoma, and synovial sarcoma (44). Sentinel lymph node biopsy is part of the standard procedure for several malignancies such as breast cancer and melanoma, in which the absence of metastatic cells in the sentinel lymph nodes would render further nodal involvement unlikely because they are the first lymph nodes to receive lymphatic drainage from the tumor. Alcorn et al. investigated the utility of sentinel lymph node biopsy and its concordance with imaging studies. They found that positive sentinel node biopsy did not necessarily correspond to findings on imaging studies such as enlarged lymph nodes on CT scans and positivity on PET scans (45). Andreou et $a l$. reported that sentinel lymph node biopsy was useful for patients with clear cell sarcoma, which was able to detect clinically occult regional lymph node metastases successfully (46). On the other hand, they found that sentinel lymph node biopsy appeared to be of little benefit for patients with synovial sarcoma. Whether sentinel lymph node biopsy for soft tissue sarcomas can help improve outcomes is still not fully established, and further work is needed to define which patients and histologic subtypes with extremity sarcomas would benefit from the procedure.

\section{Soft tissue sarcomas after unplanned excision ("Whoops" surgery)}

Unplanned excision is defined as tumor resection without preoperative diagnostic examinations and without the intention to achieve microscopically negative margins (47). Soft tissue sarcomas have a greater risk of being treated by unplanned surgery because the incidence of soft tissue sarcoma is too low for most clinicians to have adequate experience managing the disease (48). The biggest problem of unplanned excision is contamination by the tumor cells, spreading into the surgical area and the surrounding tissue. Macroscopic residual tumor is often present after the unplanned excision (49). It is therefore not surprising that unplanned excisions are associated with an increased risk of local recurrence unless followed by tumor bed excision (50). At re-resection surgery after the unplanned excision, the goal should be to remove the whole surgical bed including areas of skin incision, surgical drain tract, and postoperative hematoma (51). Transverse or oblique incisions at the unplanned excision may result in contamination of multiple or adjacent compartments, which may necessitate a much more extensive resection to achieve microscopically negative margins. In such cases, reconstruction of soft tissue with a flap is frequently required. 


\section{Recommendations}

\section{Initial assessment and treatment planning}

The initial assessment of patients with a soft tissue mass should start with a thorough documentation of the patient's history, followed by physical examination, including inspection and palpation. In order to visualize the soft tissue mass and the extent of invasion around the primary site, contrast-enhanced MRI is the most useful imaging modality, especially for invasive soft tissue sarcomas. In the diagnosis of soft tissue tumors, biopsy is an essential test for determining malignancy, histological subtype, and grade. For the patients diagnosed with a soft tissue sarcoma, essential imaging would include baseline chest X-ray and CT scan. Other tests, including ultrasound of lymph nodes, CT of abdomen and pelvis, bone scan, and PET-CT scan may be appropriate for certain histologic subtypes and stages.

For tumors that are sensitive to chemotherapy such as rhabdomyosarcoma and extraskeletal Ewing sarcoma, preoperative chemotherapy has been the standard of care. Preoperative chemotherapy may also be considered for some high-grade non-round cell soft tissue sarcomas that are relatively sensitive to chemotherapy, such as myxoid liposarcoma, especially if they are large in size $(>10 \mathrm{~cm})$. For tumors that cannot be expected to benefit from chemotherapy or radiotherapy, or elderly people who have difficulty in tolerating chemotherapy, it may be preferable to focus on local treatment with surgery and radiation.

\section{Surgical planning}

The surgeon should take into consideration the histological diagnosis, the anatomy at the site of disease, the speed of tumor growth, and the general condition of the patient, before determining the area of resection and the reconstructive method. At the very outset, it is very important to clarify the purpose of the surgery ahead of surgical planning. Surgical treatment is usually done with cure of the patient in mind, and the objective is to eliminate completely the sarcoma from the extremity; however, sometimes surgery is also done for the purpose of improving quality of life and alleviating symptoms. In cases done with curative intent, it is a top priority to secure sufficiently wide margins and to achieve, at a minimum, microscopically negative margins (R0 resection). In cases performed for palliation, relief of symptoms is given greater priority (52). It is important to stress that even in palliative surgery, local control of disease remains an important goal, and local relapse or progression can be especially miserable for a terminal patient.

In the preoperative planning of a curative surgery, the resection area is set in principle so as to obtain a microscopically negative margin based on image findings, particularly the contrast enhanced MRI scan. Because soft tissue sarcomas occur in all parts of the body, the anatomic conditions are vastly different for each case. In order to attain the targeted resection margins, it is necessary to decide carefully which healthy tissues in the surroundings should be removed. For most soft tissue sarcomas, it is justifiable to plan to resect a tumor with a margin of 1 to $2 \mathrm{~cm}$ of muscle or fat; it may also be acceptable to resect less tissue if there is a durable barrier, such as a layer of thick fascia or periosteum. However, a margin of $3 \mathrm{~cm}$ or more is potentially required in the planning for highly invasive soft tissue sarcoma, such as superficial spreading myxofibrosarcoma. Even if the pathological diagnosis of the biopsy is low-grade, the temptation to reduce the extent of resection should be avoided as it may increase the risk of local recurrence. Histological grade reflects metastatic potential of the tumor more than local aggressiveness (53).

It is of course important to keep in mind the value of preserving body function. If the tumor is close to critical structures such as blood vessels, nerves, bones, ligaments, and tendons, adjuvant therapy such as preoperative or postoperative radiotherapy and/or chemotherapy should be considered. For the patients receiving preoperative chemotherapy and/or radiotherapy, an image examination should be done after completion of preoperative treatment, and preoperative planning should be based on the images obtained immediately before the surgery. If preoperative radiotherapy and/or chemotherapy has been effective and the tumor has shrunk, the extent of surgical resection might be reduced (54). On the other hand, if the preoperative treatment has been ineffective and the tumor has grown, it may be necessary to expand the range of resection and perhaps even perform amputation.

Currently, limb-sparing surgery is the mainstay of surgical treatment for most soft tissue sarcomas. The limbsparing rate has exceeded $90 \%$ in recent years. This has been accomplished by the development of better diagnostic imaging, superior techniques for limb reconstruction, and the application of perioperative radiotherapy and/or chemotherapy $(55,56)$. Limb-sparing surgery with a wide margin is generally preferred because it can achieve a low rate of local recurrence with an acceptable level of morbidity (57). Amputation of limbs may be necessary when the tumor has 
infiltrated into major blood vessels, nerves, or other critical structures, particularly if the tumor is of a massive size and the loss of muscle mass be prohibitive to meaningful postoperative function. In the surgical planning of a curative surgery, tumor resection with macroscopic positive margins (R2 resection) is not permissible for any reasons. After determining the range of resection, the reconstructive surgery should be tailored to address the tissue defects. The converse is to be discouraged strongly. The extent of resection must not be compromised or reduced in order to make the reconstructive surgery easier.

\section{Notes on tumor resection}

A critical aspect of surgery for soft tissue sarcoma is adherence to the planned cutting edge of resection. Since the surgical margin adopts the smallest margin in the resected specimen, cutting into the planned resection area is prohibited. Of particular note, subcutaneous tissue and muscle tissue may contract, deform, or move during surgery, so it is necessary to perform surgery carefully with frequent confirmation of the resection plane. Moreover, since the subcutaneous tissues are prone to detach from the fascia and expose the biopsy tract or even the tumor itself, it is sometime prudent to stitch together the fascia to the skin and subcutaneous fat so as not to allow these tissues to peel off.

\section{Intraoperative frozen section diagnosis}

The rationale for intraoperative pathological assessment includes verification of sample adequacy at the time of biopsy, confirmation of diagnosis prior to definite surgery, and assessment of surgical margins. The latter application is often helpful for resection of certain soft tissue sarcomas, particularly those which are invasive. Examples would include myxofibrosarcoma, dermatofibrosarcoma protuberans (DFSP), and desmoid tumors. These tumors are notorious for extending microscopically far beyond the visible and palpable mass. On occasion, a positive frozen section would alert the surgeon to resect more tissue in an area not obviously affected. It is also justifiable to submit for frozen section specimens of tissue that appear abnormal or suspicious for a given anatomic area. However, surgeons should appreciate the limitations of intraoperative frozen sections. They cannot supplant the definitive margin assessment on final pathology, and a negative frozen section margin does not guarantee a negative final margin. Random sampling of tissues in most cases is not a useful or cost- effective practice; rather, surgeons must judiciously select the tissues to submit, such as fascia for superficial spreading sarcomas and sub-dermal tissue for DFSP. Definitive histologic diagnosis is generally not possible with frozen section, and it should not be used to discriminate between similar-appearing lesions, such as benign lipoma and atypical lipomatous tumor. Finally, assessment of surgical margin of invasive spindle cell sarcomas can be difficult because sparse proliferation of spindle tumor cells can be difficult to distinguish from normal fibroblasts in the fascia and other connective tissue. Intraoperative frozen section diagnosis is better able to detect a positive margin when the tumor cells show exuberant proliferation at the surgical margins.

\section{Assessment of surgical margins}

Resected specimens of soft tissue sarcoma can easily change shape. The surrounding connective tissues, especially muscle fibers, frequently become shortened because of formalin fixation and a decrease in tension. Assessment of surgical margins of soft tissue sarcoma requires special care. Surgical margins of the resected specimen should be examined and documented by both the surgeon and the pathologist together to avoid misunderstanding (58). Application of ink on the surface of the surgical resection is recommended to evaluate the surgical margin precisely (59). If necessary, temporary stabilization of soft tissues with pins, a rubber plate, or other devices are useful to avoid change in shape of the resected specimens.

\section{Adjuvant therapy when surgical margin turns out positive}

The National Comprehensive Cancer Network (NCCN) Guidelines and ESMO-EURACAN Clinical Practice Guidelines state that when surgical resection margins are positive on final pathology (other than bone, nerve, or major blood vessels), surgical re-resection aiming at microscopically negative (R0) margin should be considered if feasible $(58,60)$. If $\mathrm{R} 0$ resection is not feasible, it is recommended that radiotherapy to the surgical field be considered. In the randomized prospective study of adjuvant radiation therapy for soft tissue sarcomas of the extremities, local recurrence was significantly related to the absence of radiotherapy; local recurrence occurred in 1 of the 70 patients with radiotherapy $(1.4 \%)$ and 17 of the 71 patients without radiotherapy (23.9\%) (61). Notably, this study included patients with both positive and negative margins (61). Alektiar et al. investigated patients with microscopically margin-positive high-grade soft 
tissue sarcoma and found that the 5-year local control rate was significantly higher in the radiotherapy group compared to the no radiotherapy group (74\% vs. 56\%, respectively) (62). Jebsen et al. surveyed adult patients with extremities and trunk wall soft tissue sarcoma treated at Scandinavian Sarcoma Group hospitals and showed that adjuvant radiotherapy reduced local recurrence in patients, irrespective of the type of margin (63). In their cohort, the risk of local recurrence was higher in the patients with intralesional and marginal margins compared to wide and radical margins (63). It should be noted that while postoperative radiotherapy improves local control, it cannot reduce the local recurrence rate to a level comparable to that of patients with negative margins when the radiotherapy is applied to the patients with frankly positive margins.

\section{Summary}

Curative surgery for extremity soft tissue sarcomas should aim for at least an $\mathrm{R} 0$ resection. If achieving $\mathrm{R} 0$ margins is thought to be difficult during preoperative planning, preoperative radiotherapy would be an option. Postoperative radiotherapy or re-resection aiming at microscopically negative margin should be considered when the resected margin turns out microscopically positive on histological examination. It is still unclear how much distance from the tumor to the planned resection plane on preoperative imaging is required to obtain R0 margin. This may vary with histologic subtype. For highly invasive soft tissue sarcomas such as myxofibrosarcoma, resection with fascial margins of $3 \mathrm{~cm}$ may not reliably achieve negative $\mathrm{R} 0$ margins. In such cases, the surgeon must be prepared to resect an even greater area, if feasible, and/or use intra-operative frozen sections to help guide the extent of tissue removal. Surgical management with appropriate margins improves local disease control and potentially increases patients' survival.

\section{Acknowledgements}

None.

\section{Footnote}

Conflicts of Interest: The authors have no conflicts of interest to declare.

\section{References}

1. Hoang K, Gao Y, Miller BJ. The Variability in Surgical
Margin Reporting in Limb Salvage Surgery for Sarcoma. Iowa Orthop J 2015;35:181-6.

2. Enneking WF, Spanier SS, Goodman MA. A system for the surgical staging of musculoskeletal sarcoma. Clin Orthop Relat Res 1980;(153):106-20.

3. Trovik CS, Bauer HC, Alvegard TA, et al. Surgical margins, local recurrence and metastasis in soft tissue sarcomas: 559 surgically-treated patients from the Scandinavian Sarcoma Group Register. Eur J Cancer 2000;36:710-6.

4. Trovik CS, Skjeldal S, Bauer H, et al. Reliability of Margin Assessment after Surgery for Extremity Soft Tissue Sarcoma: The SSG Experience. Sarcoma 2012;2012:290698.

5. AJCC Manual for Staging of Cancer. 3rd ed. Philadelphia: JB Lippincott, 1988.

6. Wittekind C, Compton CC, Greene FL, et al. TNM residual tumor classification revisited. Cancer 2002;94:2511-6.

7. Kainhofer V, Smolle MA, Szkandera J, et al. The width of resection margins influences local recurrence in soft tissue sarcoma patients. Eur J Surg Oncol 2016;42:899-906.

8. Gundle KR, Kafchinski L, Gupta S, et al. Analysis of Margin Classification Systems for Assessing the Risk of Local Recurrence After Soft Tissue Sarcoma Resection. J Clin Oncol 2018;36:704-9.

9. Rodriguez-Galindo C, Shah N, McCarville MB, et al. Outcome after local recurrence of osteosarcoma: the St. Jude Children's Research Hospital experience (1970-2000). Cancer 2004;100:1928-35.

10. Dickinson IC, Whitwell DJ, Battistuta D, et al. Surgical margin and its influence on survival in soft tissue sarcoma. ANZ J Surg 2006;76:104-9.

11. Novais EN, Demiralp B, Alderete J, et al. Do surgical margin and local recurrence influence survival in soft tissue sarcomas? Clin Orthop Relat Res 2010;468:3003-11.

12. Vraa S, Keller J, Nielsen OS, et al. Soft-tissue sarcoma of the thigh: surgical margin influences local recurrence but not survival in 152 patients. Acta Orthop Scand 2001;72:72-7.

13. Gronchi A, Miceli R, Fiore M, et al. Extremity soft tissue sarcoma: adding to the prognostic meaning of local failure. Ann Surg Oncol 2007;14:1583-90.

14. Potter BK, Hwang PF, Forsberg JA, et al. Impact of margin status and local recurrence on soft-tissue sarcoma outcomes. J Bone Joint Surg Am 2013;95:e151.

15. Ahmad R, Jacobson A, Hornicek F, et al. The Width of the Surgical Margin Does Not Influence Outcomes in 
Extremity and Truncal Soft Tissue Sarcoma Treated With Radiotherapy. Oncologist 2016;21:1269-76.

16. Tang YW, Lai CS. The significance of close but negative excision margin for treatment of soft-tissue sarcoma. Ann Plast Surg 2012;69:633-6.

17. Heslin MJ, Woodruff J, Brennan MF. Prognostic significance of a positive microscopic margin in highrisk extremity soft tissue sarcoma: implications for management. J Clin Oncol 1996;14:473-8.

18. Stojadinovic A, Leung DH, Hoos A, et al. Analysis of the prognostic significance of microscopic margins in 2,084 localized primary adult soft tissue sarcomas. Ann Surg 2002;235:424-34.

19. Tanabe KK, Pollock RE, Ellis LM, et al. Influence of surgical margins on outcome in patients with preoperatively irradiated extremity soft tissue sarcomas. Cancer 1994;73:1652-9.

20. Bonvalot S, Levy A, Terrier P, et al. Primary Extremity Soft Tissue Sarcomas: Does Local Control Impact Survival? Ann Surg Oncol 2017;24:194-201.

21. Yoo HJ, Hong SH, Kang Y, et al. MR imaging of myxofibrosarcoma and undifferentiated sarcoma with emphasis on tail sign; diagnostic and prognostic value. Eur Radiol 2014;24:1749-57.

22. Kikuta K, Kubota D, Yoshida A, et al. An analysis of factors related to recurrence of myxofibrosarcoma. Jpn J Clin Oncol 2013;43:1093-104.

23. Haglund KE, Raut CP, Nascimento AF, et al. Recurrence patterns and survival for patients with intermediate- and high-grade myxofibrosarcoma. Int J Radiat Oncol Biol Phys 2012;82:361-7.

24. Ghazala CG, Agni NR, Ragbir M, et al. Myxofibrosarcoma of the extremity and trunk: a multidisciplinary approach leads to good local rates of LOCAL control. Bone Joint J 2016;98-B:1682-8.

25. Iwata S, Yonemoto T, Araki A, et al. Impact of infiltrative growth on the outcome of patients with undifferentiated pleomorphic sarcoma and myxofibrosarcoma. J Surg Oncol 2014;110:707-11.

26. Evans HL, Soule EH, Winkelmann RK. Atypical lipoma, atypical intramuscular lipoma, and well differentiated retroperitoneal liposarcoma: a reappraisal of 30 cases formerly classified as well differentiated liposarcoma. Cancer 1979;43:574-84.

27. Mussi CE, Daolio P, Cimino M, et al. Atypical lipomatous tumors: should they be treated like other sarcoma or not? Surgical consideration from a bi-institutional experience. Ann Surg Oncol 2014;21:4090-7.
28. Bishop AJ, Zagars GK, Torres KE, et al. Combined Modality Management of Retroperitoneal Sarcomas: A Single-Institution Series of 121 Patients. Int J Radiat Oncol Biol Phys 2015;93:158-65.

29. Ferguson PC, Griffin AM, O'Sullivan B, et al. Bone invasion in extremity soft-tissue sarcoma: impact on disease outcomes. Cancer 2006;106:2692-700.

30. Lin PP, Pino ED, Normand AN, et al. Periosteal margin in soft-tissue sarcoma. Cancer 2007;109:598-602.

31. Puhaindran ME, Rohde RS, Chou J, et al. Clinical outcomes for patients with soft tissue sarcoma of the hand. Cancer 2011;117:175-9.

32. Brien EW, Terek RM, Geer RJ, et al. Treatment of soft-tissue sarcomas of the hand. J Bone Joint Surg Am 1995;77:564-71.

33. Pradhan A, Cheung YC, Grimer RJ, et al. Soft-tissue sarcomas of the hand: oncological outcome and prognostic factors. J Bone Joint Surg Br 2008;90:209-14.

34. Puhaindran ME, Steensma MR, Athanasian EA. Partial hand preservation for large soft tissue sarcomas of the hand. J Hand Surg Am 2010;35:291-5.

35. Puhaindran ME, Athanasian EA. Double ray amputation for tumors of the hand. Clin Orthop Relat Res 2010;468:2976-9.

36. Lin PP, Guzel VB, Pisters PW, et al. Surgical management of soft tissue sarcomas of the hand and foot. Cancer 2002;95:852-61.

37. Rohde RS, Puhaindran ME, Morris CD, et al. Complications of radiation therapy to the hand after soft tissue sarcoma surgery. J Hand Surg Am 2010;35:1858-63.

38. Bray PW, Bell RS, Bowen CV, et al. Limb salvage surgery and adjuvant radiotherapy for soft tissue sarcomas of the forearm and hand. J Hand Surg Am 1997;22:495-503.

39. Jyothirmayi R, Sittampalam Y, Harmer C. Soft tissue sarcoma of the hand or foot: conservative surgery and radiotherapy. Sarcoma 1999;3:17-24.

40. Talbert ML, Zagars GK, Sherman NE, et al. Conservative surgery and radiation therapy for soft tissue sarcoma of the wrist, hand, ankle, and foot. Cancer 1990;66:2482-91.

41. Colterjohn NR, Davis AM, O'Sullivan B, et al. Functional outcome in limb-salvage surgery for soft tissue tumours of the foot and ankle. Sarcoma 1997;1:67-74.

42. Cribb GL, Loo SC, Dickinson I. Limb salvage for softtissue sarcomas of the foot and ankle. J Bone Joint Surg Br 2010;92:424-9.

43. Nishimura A, Matsumine A, Asanuma K, et al. The adverse effect of an unplanned surgical excision of foot soft tissue sarcoma. World J Surg Oncol 2011;9:160. 
44. Keung EZ, Chiang YJ, Voss RK, et al. Defining the incidence and clinical significance of lymph node metastasis in soft tissue sarcoma. Eur J Surg Oncol 2018;44:170-7.

45. Alcorn KM, Deans KJ, Congeni A, et al. Sentinel lymph node biopsy in pediatric soft tissue sarcoma patients: utility and concordance with imaging. J Pediatr Surg 2013;48:1903-6.

46. Andreou D, Boldt H, Werner M, et al. Sentinel node biopsy in soft tissue sarcoma subtypes with a high propensity for regional lymphatic spread--results of a large prospective trial. Ann Oncol 2013;24:1400-5.

47. Noria S, Davis A, Kandel R, et al. Residual disease following unplanned excision of soft-tissue sarcoma of an extremity. J Bone Joint Surg Am 1996;78:650-5.

48. Randall RL, Bruckner JD, Papenhausen MD, et al. Errors in diagnosis and margin determination of softtissue sarcomas initially treated at non-tertiary centers. Orthopedics 2004;27:209-12.

49. Giuliano AE, Eilber FR. The rationale for planned reoperation after unplanned total excision of soft-tissue sarcomas. J Clin Oncol 1985;3:1344-8.

50. Morii T, Yabe H, Morioka H, et al. Clinical significance of additional wide resection for unplanned resection of high grade soft tissue sarcoma. Open Orthop J 2008;2:126-9.

51. Pretell-Mazzini J, Barton MD, Jr., Conway SA, et al. Unplanned excision of soft-tissue sarcomas: current concepts for management and prognosis. J Bone Joint Surg Am 2015;97:597-603.

52. Sheldon DG, James TA, Kraybill WG. Palliative surgery of soft tissue sarcoma. Surg Oncol Clin N Am 2004; 13:531-41, ix.

53. Biau DJ, Ferguson PC, Chung P, et al. Local recurrence of localized soft tissue sarcoma: a new look at old predictors. Cancer 2012;118:5867-77.

54. Dagan R, Indelicato DJ, McGee L, et al. The significance of a marginal excision after preoperative radiation therapy for soft tissue sarcoma of the extremity. Cancer 2012;118:3199-207.

55. Ghert MA, Davis AM, Griffin AM, et al. The surgical and functional outcome of limb-salvage surgery with vascular reconstruction for soft tissue sarcoma of the extremity. Ann Surg Oncol 2005;12:1102-10.

56. Steinau HU, Daigeler A, Langer S, et al. Limb salvage in malignant tumors. Semin Plast Surg 2010;24:18-33.

57. Williard WC, Hajdu SI, Casper ES, et al. Comparison of amputation with limb-sparing operations for adult soft tissue sarcoma of the extremity. Ann Surg 1992;215:269-75.

58. NCCN Clinical Practice Guidelines in Oncology Soft Tissue Sarcoma Version 2.2018.

59. Docquier PL, Paul L, Cartiaux O, et al. Formalin fixation could interfere with the clinical assessment of the tumorfree margin in tumor surgery: magnetic resonance imaging-based study. Oncology 2010;78:115-24.

60. Casali PG, Abecassis N, Bauer S, et al. Soft tissue and visceral sarcomas: ESMO-EURACAN Clinical Practice Guidelines for diagnosis, treatment and follow-up. Ann Oncol 2018. [Epub ahead of print].

61. Yang JC, Chang AE, Baker AR, et al. Randomized prospective study of the benefit of adjuvant radiation therapy in the treatment of soft tissue sarcomas of the extremity. J Clin Oncol 1998;16:197-203.

62. Alektiar KM, Velasco J, Zelefsky MJ, et al. Adjuvant radiotherapy for margin-positive high-grade soft tissue sarcoma of the extremity. Int J Radiat Oncol Biol Phys 2000;48:1051-8.

63. Jebsen NL, Trovik CS, Bauer HC, et al. Radiotherapy to improve local control regardless of surgical margin and malignancy grade in extremity and trunk wall soft tissue sarcoma: a Scandinavian sarcoma group study. Int J Radiat Oncol Biol Phys 2008;71:1196-203.
Cite this article as: Endo M, Lin PP. Surgical margins in the management of extremity soft tissue sarcoma. Chin Clin Oncol 2018;7(4):37. doi: 10.21037/cco.2018.08.10 